\title{
AN EXTREMUM PROBLEM FOR ANALYTIC FUNCTIONS WITH AREA NORM
}

\author{
EDGAR REICH ${ }^{1}$
}

0. Introduction. Let $\mathscr{K}$ be the class of complex valued, bounded, measurable functions $x(z), z \in U=\{|z|<1\}$. Our principal objective is to attempt to determine whether for a given function $x(z), x \in \mathscr{K}$, strict inequality holds in the obvious inequality,

$$
\sup _{\substack{f \in \mathscr{B} \\ f \neq 0}} \frac{\left|\iint_{U} x(z) f(z) d x d y\right|}{\iint|f(z)| d x d y} \leqq\|x\|_{\infty}=\operatorname{ess} \sup _{z \in U}|x(z)| .
$$

The class $\mathscr{B}$ over which the sup on the left side is taken is the Banach space of functions $f$ holomorphic in $U$ for which

$$
\|f\|=\int_{U}|f(z)| d x d y<\infty .
$$

If equality holds in $(0.1)$ we say $x \in \mathscr{K}^{*}$.

Because the norm for $\mathscr{B}$ involves $|f(z)|$ to the first power the problem of determining whether a given $\varkappa$ belongs to $\mathscr{K}^{*}$ or $\mathscr{K} \backslash \mathscr{K}^{*}$ turns out to be rather delicate. We succeed in determining certain useful necessary conditions as well as certain sufficient conditions. Aside from its function theoretic interest there exists an intimate connection of our problem with a problem of the theory of plane quasiconformal mappings that was originally formulated by Teichmüller $[12, \mathrm{p} .184]$ : To characterize those quasiconformal mappings of $U$ onto $U$ possessing a given boundary correspondence which are "extremal" in the sense of having minimal maximal dilatation. It is now known [2], [8] that a quasiconformal mapping of $U$ is

\footnotetext{
1 Work done with support from National Science Foundation grant Grant GP. $27275 \mathrm{~A} 2$.
} 
extremal for its boundary correspondence if and only if its complex dilatation $x(z)$ belongs to the class $\mathscr{K}^{*}$. This fact allows a "geometric" attack on our question which we however deliberately choose to avoid in the present paper precisely in order to try to enlarge the available analytic techniques for the problem.

The following notation will be followed.

$$
\begin{gathered}
L_{\varkappa}[f]=\int_{U} \int_{U} x(z) f(z) d x d y, \\
\left\|L_{\varkappa}\right\|=\sup _{\substack{f \in \mathscr{B} \\
f \neq 0}} \frac{\left|L_{\varkappa}[f]\right|}{\|f\|}, \\
\mathscr{N}=\left\{x: x \in \mathscr{K}, L_{\varkappa}[f]=0 \text { for all } f \in \mathscr{B}\right\}, \\
\mathscr{K}_{T}=\left\{x: \varkappa \in \mathscr{K}, x=k \frac{\varphi(z)}{|\varphi(z)|} \text { where } k=\text { const }>0, \varphi \in \mathscr{B}\right\} .
\end{gathered}
$$

It is clear that $\mathscr{K}_{T} \subset K^{*}$. Only if $\varkappa \in \mathscr{K}_{T}$ can there exist a function $f_{0} \in \mathscr{B}$ with $\|\chi\|_{\infty}=\left|L_{\varkappa}\left[f_{0}\right]\right| /\left\|f_{0}\right\|$. As was first shown by Strebel [9], however, $\mathscr{K}^{*} \backslash \mathscr{K}_{T}$ is nonempty; it is precisely this fact that makes our problem non trivial.

Example 0.1.2 In

$$
\begin{aligned}
\Omega=\left\{w=u+i v: 0<v<u^{\alpha}, 0<u<A\right\} & \\
& (\alpha>1,0<A \leqq \infty)
\end{aligned}
$$

choose $\mu(w)$ such that

$$
|\mu(w)| \leqq 1, \quad \lim _{\substack{w \rightarrow 0 \\ w \in \Omega}} \mu(w)=1 .
$$

Let

$$
g_{n}(w)=\frac{n^{\alpha+1}}{\Gamma(\alpha+1)} e^{-n w}, \quad w \in \Omega .
$$

Let $w=\Phi(z)$ map $U$ conformally onto $\Omega$, and define $\varkappa(z), f_{n}(z), z \in U$, by

$$
\varkappa(z) \frac{\overline{d z}}{d z}=\mu(w) \frac{\overline{d w}}{d w}, \quad(w=\Phi(z))
$$

\footnotetext{
2 The above example is related to an example in [4].
} 


$$
f_{n}(z) d z^{2}=g_{n}(w) d w^{2}, \quad(w=\Phi(z)),
$$

respectively. We obtain

$$
\left\|f_{n}\right\|=\iint_{\Omega}\left|g_{n}(w)\right| d u d v \rightarrow 1, \quad L_{\varkappa}\left[f_{n}\right]=\iint_{\Omega} \mu(w) g_{n}(w) d u d v \rightarrow 1,
$$

and

$$
|\varkappa(z)|=|\mu(\Phi(z))| \leqq 1, \quad z \in U .
$$

Since $\left\|L_{\varkappa}\right\|=\|\varkappa\|_{\infty}=1$ we have $\varkappa \in \mathscr{K}^{*}$, but if $|\mu(w)|$ is not constant, or if $\mu(w)=1$ but $A=\infty, x$ will not belong to $\mathscr{K}_{T}$.

We shall say that a sequence $\varphi_{n} \in \mathscr{B}$ is an extremal sequence for $x$ if $\left\|\varphi_{n}\right\|=1, \quad n=1,2, \ldots$, and

$$
L_{\varkappa}\left[\varphi_{n}\right] \rightarrow\left\|L_{\varkappa}\right\|
$$

Since the subclass of $\mathscr{B}$ of functions with norm not exceeding a fixed constant is a normal family we can assume (if necessary by choosing a subsequence) that

$$
\varphi_{n}(z) \rightarrow \varphi_{0}(z) \quad \text { locally uniformly in } U \text {. }
$$

$\varphi_{0}$ will be called a limit function for $\varkappa$. If $\varphi_{0}(z)$ vanishes identically the extremal sequence is said to degenerate.

$\mathrm{L}$ e m m a 0.1. $x \in \mathscr{K} \backslash \mathscr{K}^{*}$ if and only if there exists $a y \in \mathscr{N}$ such that

$$
\|x-v\|_{\infty}<\|x\|_{\infty} .
$$

Proof. (i) If $(0.6)$ holds then $\left\|L_{x}\right\|=\left\|L_{x-v}\right\|<\|x\|_{\infty}$. Hence $x \notin \mathscr{K}^{*}$.

(ii) By the Hahn-Banach theorem and the Riesz representation theorem there exists $\tilde{x} \in \mathscr{K}$ with $\|\tilde{x}\|_{\infty}=\left\|L_{\varkappa}\right\|$ such that

$$
L_{\varkappa}[f]=L_{\tilde{\varkappa}}[f], \quad f \in \mathscr{B} .
$$

Hence $v=x-\tilde{x} \in \mathscr{N}$. So, if $\left\|L_{\varkappa}\right\|<\|x\|_{\infty},(0.6)$ will hold.

The following two lemmas deal with extremal sequences and a consequence of non-degeneracy; although they are equivalent to closely related facts found elsewhere we list them explicitly for the sake of completeness.

L e m m a 0.2. If $\mu \in \mathscr{K}^{*}$ and $\varphi_{n} \in \mathscr{B},\left\|\varphi_{n}\right\|=1$, then $\left\{\varphi_{n}(z)\right\}$ is an extremal sequence for $\mu$ if and only if

$$
\lim _{n \rightarrow \infty}\left[\iint_{S} \mu(z) \varphi_{n}(z) d x d y-\|\mu\|_{\infty} \iint_{S}\left|\varphi_{n}(z)\right| d x d y\right]=0,
$$

for every measurable subset $S$ of $U$. 
Proof. Since we can put $S=U,(0.8)$ implies that $\left\{\varphi_{n}\right\}$ is extremal sequence for $\mu$. To prove that (0.8) is necessary, consider

$$
\|\mu\|_{\infty}=z_{n}+\int_{U \backslash S} \int_{n} \varphi_{n} \mu d x d y+\delta_{n}
$$

where $z_{n}=\iint_{S} \varphi_{n} \mu d x d y$, and $\delta_{n}=\|\mu\|_{\infty}-\iint_{U} \varphi_{n} \mu d x d y \rightarrow 0$. Taking real parts of both sides,

$$
\begin{aligned}
\|\mu\|_{\infty} & \leqq \operatorname{Re} z_{n}+\|\mu\|_{\infty} \int_{U \backslash S} \int_{S}\left|\varphi_{n}\right| d x d y+\operatorname{Re} \delta_{n} \\
& =\operatorname{Re} z_{n}+\|\mu\|_{\infty}\left(1-\iint_{S}\left|\varphi_{n}\right| d x d y\right)+\operatorname{Re} \delta_{n} .
\end{aligned}
$$

Rearranging terms and taking into account that

$$
\left|z_{n}\right| \leqq\|\mu\|_{\infty} \iint_{S}\left|\varphi_{n}\right| d x d y
$$

we obtain

$$
\frac{\left|z_{n}-\operatorname{Re} z_{n}\right|^{2}}{2\left|z_{n}\right|} \leqq\left|z_{n}\right|-\operatorname{Re} z_{n} \leqq|| \mu||_{\infty} \int_{S} \int\left|\varphi_{n}\right| d x d y-\operatorname{Re} z_{n} \leqq \operatorname{Re} \delta_{n}
$$

Since $\left\{z_{n}\right\}$ is bounded, we see that $z_{n}-\operatorname{Re} z_{n} \rightarrow 0$, and (0.8) follows.

If $\varkappa$ has a non-degenerate extremal sequence a representation for $L_{x}[f]$ is obtained as follows.

L e m m a 0.3. Suppose $\varkappa \in \mathscr{K}$ has the limit function $\varphi_{0}(z)=\lim _{n \rightarrow \infty} \varphi_{n}(z)$, $\left\|\varphi_{0}\right\| \neq 0$. Then

$$
L_{\varkappa}[f]=\left\|L_{\varkappa}\right\| \iint_{U} \frac{\overline{\varphi_{0}(z)}}{\left|\varphi_{0}(z)\right|} f(z) d x d y, \quad f \in \mathscr{B} .
$$

Proof. Again choose $\tilde{\varkappa} \in \mathscr{K}$ such that

$$
L_{\tilde{\varkappa}}[f]=L_{\varkappa}[f], \quad f \in \mathscr{B}, \quad\|\tilde{\varkappa}\|_{\infty}=\left\|L_{\varkappa}\right\| .
$$

In Lemma 0.2 choose $S=\{R<|z|<1\}$. Thus

$$
\iint_{R<|z|<1} \varphi_{n} \tilde{x} d x d y-\|\tilde{x}\|_{\infty}\left(1-\int_{|\xi|<R}\left|\varphi_{n}\right| d x d y\right) \rightarrow 0,
$$

that is 


$$
\begin{aligned}
\int_{R<|z|<1} \int_{n} \varphi_{n} \tilde{x} d x d y & \rightarrow\|\tilde{x}\|_{\infty}\left(1-\iint_{|z|<R}\left|\varphi_{0}\right| d x d y\right) \\
& =\|\tilde{x}\|_{\infty}\left(1-\left\|\varphi_{0}\right\|+\iint_{R<|z|<1}\left|\varphi_{0}\right| d x d y\right) .
\end{aligned}
$$

Therefore,

$$
\begin{aligned}
& L_{\varkappa}\left[\varphi_{n}-\varphi_{0}\right]=L_{\varkappa}\left[\varphi_{n}-\varphi_{0}\right] \\
& =\iint_{|z|<R}\left(\varphi_{n}-\varphi_{0}\right) \tilde{\varkappa} d x d y+\iint_{R<|z|<1}\left(\varphi_{n}-\varphi_{0}\right) \tilde{\varkappa} d x d y \rightarrow l(R) \\
& =0+\|\tilde{\varkappa}\|_{\infty}\left(1-\| \varphi_{0}||+\iint_{R<|z|<1}\left|\varphi_{0}\right| d x d y\right)-\iint_{R<|z|<1} \varphi_{0} \tilde{x} d x d y .
\end{aligned}
$$

Hence, $l(R)$ is independent of $R$; in particular, $l(0)=l(1-0)$; that is,

$$
\|\tilde{\varkappa}\|_{\infty}\left\|\varphi_{0}\right\|=L_{\tilde{\varkappa}}\left[\varphi_{0}\right] .
$$

This implies (0.9).

An immediate corollary of Lemma 0.3 is the following: Suppose $x \in \mathscr{K}^{*}$. Then either every extremal sequence for $x$ degenerates or $\varkappa \in \mathscr{K}_{T}$.

If one takes $A<\infty, \mu \equiv 1$ in Example 0.1 one obtains $x \in \mathscr{K}_{T}$ with a degenerative extremal sequence. Another example was given in [7].

1. The Class $\mathscr{N}$. In order to exploit (0.6) it is necessary to build up an arsenal of functions of class $\mathscr{N}$ to be used to "combat" the $x^{\prime} s$ for the purpose of trying to reduce the latters' norms.

Since the functions analytic in the closed disk $\{|z| \leqq 1\}$ are dense in $\mathscr{B}$ in the norm \|\| , and since these in turn can be uniformly approximated by polynomials it is clear that

$$
v \in \mathscr{N} \Leftrightarrow \iint_{U} v(z) z^{n} d x d y=0, \quad n=0,1,2, \ldots,
$$

We may interpret (1.1) in terms of Fourier series: Suppose

$$
v\left(r e^{i \vartheta}\right) \sim \sum_{n=1}^{\infty} \alpha_{n}(r) e^{i n \vartheta}+\sum_{n=0}^{\infty} \beta_{n}(r) e^{-i n \vartheta}, \quad 0 \leqq r<1 .
$$

A necessary and sufficient condition for $v$ to belong to $\mathscr{N}$ is that $v \in \mathscr{K}$, and

$$
\int_{0}^{1} r^{n+1} \beta_{n}(r) d r=0, \quad n=0,1,2, \ldots, .
$$


A function of class $\mathscr{N}$ may be chosen arbitrarily on a compact subset of $U$, subject only to the restriction that it is bounded there. This is a corollary of the following result.

The or e m 1.1. Let $\alpha(z)$ be a bounded, measurable, complex valued function, $|z| \leqq \varrho,(0<\varrho<1)$. There exists a unique function $\beta(z)$, holomorphic for $\varrho<|z| \leqq \infty$, such that

$$
v(z)= \begin{cases}\alpha(z), & |z|<\varrho, \\ \beta(z), & \varrho<|z|<1,\end{cases}
$$

belongs to $\mathscr{N}$; namely

(1.4) $\beta(z)=-\frac{1}{1-\varrho^{2}} z P(z), \quad P(z)=-\frac{1}{\pi} \int_{|\zeta|<\varrho} \int_{\zeta-z} \frac{\alpha(\zeta)}{\zeta-z} d \eta, \varrho<|z|<\infty$.

Proof. Suppose $\beta(z)$ as required exists. Then for any function $f(z)$ holomorphic for $|z| \leqq 1$, and any $\varrho_{1}, \varrho<\varrho_{1}<1$,

$$
\begin{aligned}
\iint_{\varrho_{1}<|z|<1} \beta(z) f(z) d x d y & \left.=\int_{\varrho_{1}<|z|<1} \int_{\frac{\partial}{\partial \bar{z}}} \frac{\partial}{\bar{z}} \beta f\right) d x d y \\
& =\frac{1}{2 i} \int_{|z|=1} \bar{z} \beta f d z-\frac{1}{2 i} \int_{|z|=\varrho_{1}} \bar{z} \beta f d z \\
& =\frac{1-\varrho_{1}^{2}}{2 i} \int_{C} \beta f \frac{d z}{z},
\end{aligned}
$$

where $C$ is homologous to $\left\{|z|=\varrho_{1}\right\}$ in $\{\varrho<|z|<1\}$. Hence

$$
\begin{aligned}
\frac{1-\varrho^{2}}{2 i} \int_{C} \beta f \frac{d z}{z} & =\int_{\varrho<|z|<1} \int_{\mid<f} \beta f d x d y=-\int_{|z|<\varrho} \int_{|z|<\varrho} \alpha f d x d y \\
& =-\int_{\mid<} P_{z} f d x d y=-\iint_{|z|<\varrho} \frac{\partial}{\partial \bar{z}}(P f) d x d y \\
& =-\frac{1}{2 i} \int_{|z|=\varrho} P f d z=-\frac{1}{2 i} \int_{C} P f d z .
\end{aligned}
$$

In view of the arbitrariness of $f$ it follows that

$$
\frac{1-\varrho^{2}}{z} \beta(z)+P(z)
$$

has a holomorphic extension to $U$. But $P(z)$ is holomorphic for $\varrho<|z| \leqq \infty, P(\infty)=0$, and, by hypothesis, $\beta(z)$ is holomorphic for $\varrho<|z| \leqq \infty$. Therefore, by Liouville's theorem, 


$$
\frac{1-\varrho^{2}}{z} \beta(z)+P(z) \equiv 0,
$$

and so (1.4) must hold. Conversely, suppose $\beta(z)$ is defined by (1.4). $P(z)$ is continuous in the extended plane, $P(\infty)=0$, and therefore $\beta(z)$ is bounded and holomorphic for $\varrho<|z| \leqq \infty$. By reversing the reasoning used above we conclude that for any $f(z)$ holomorphic for $|z| \leqq 1$,

$$
\iint_{\varrho<|z|<1} \beta f d x d y=-\iint_{|z|<\varrho} \alpha f d x d y \text {. }
$$

Thus $v \in \mathscr{N}$.

Remark. One sees from the proof of Theorem 1.1, or directly from condition (1.2) that to obtain $v \in \mathscr{N}$ it is sufficient to take

$$
\beta(z)=-\frac{1}{1-\varrho^{2}} z F(z), \quad \varrho<|z|<1,
$$

where $F(z)$ is any function bounded in $U$ such that

$$
F_{\bar{z}}= \begin{cases}\alpha(z), & |z|<\varrho, \\ 0, & \varrho<|z|<1,\end{cases}
$$

in the sense of distributions. Such functions $F(z)$ differ from $P(z)$ by a function bounded and holomorphic in $U$. The following necessary condition for $x \in \mathscr{K}^{*}$ follows.

Theorem 1.2. Suppose $\varkappa(z) \in \mathscr{K}^{*}$. Choose $\varrho, 0<\varrho<1$, and choose $F \in \mathscr{K}$ such that

$$
F_{\vec{z}}= \begin{cases}x(z), & |z|<\varrho, \\ 0, & \varrho<|z|<1 .\end{cases}
$$

Then for any complex number $t$ satisfying

$$
\operatorname{Re} t>\frac{1-\varrho^{2}}{2}|t|^{2}
$$

we have

$$
\sup _{\varrho<|z|<1}|\varkappa(z)+t z F(z)| \geqq\|x\|_{\infty} .
$$

Proof. By (1.5), the function

$$
v(z)= \begin{cases}\left(1-\varrho^{2}\right) t x(z), & |z|<\varrho, \\ -t z F(z), & \varrho<|z|<1,\end{cases}
$$

belongs to $\mathscr{N}$. Therefore 


$$
L_{\varkappa-\nu}[f]=L_{\varkappa}[f], \quad f \in \mathscr{B},
$$

and

$$
\|x-v\|_{\infty} \geqq \sup _{f \in \mathscr{B}} \frac{\left|L_{\varkappa-\nu}[f]\right|}{\|f\|}=\sup _{\in \mathscr{B}} \frac{\left|L_{\varkappa}[f]\right|}{\|f\|}=\|x\|_{\infty} .
$$

In view of $(1.7)$,

$$
\sup _{|z|<\varrho}|\varkappa(z)-v(z)|=\left|1-\left(1-\varrho^{2}\right) t\right| \sup _{|z|<\varrho}|\varkappa(z)|<\sup _{|z|<\varrho}|\varkappa(z)| .
$$

Therefore

$$
\sup _{\varrho<|z|<1}|x(z)+t z F(z)|=\|x-v\|_{\infty} \geqq\|x\|_{\infty} .
$$

Note. The qualitative significance of (1.8) is to restrict the absolute value and argument of $x(z)$, for $\varrho<|z|<1$, as related to the behavior of $x(z)$ for $|z|<\varrho$. Choosing $t=1 / n, n \rightarrow \infty$, we obtain the known [2] result

$$
x \in \mathscr{K}^{*}, \quad 0<\varrho<1 \Rightarrow \sup _{\varrho<|z|<1}|x(z)|=\|x\|_{\infty} \cdot^{3}
$$

We shall require a generalization of the class $\mathscr{N}$ that is obtained by replacing $U$ by an arbitrary simply connected region $\Omega$. We set

$$
\begin{gathered}
\mathscr{K}(\Omega)=\left\{\mu(w): \mu \text { is complex valued, }\|\mu\|_{\infty}=\underset{w \in \Omega}{\operatorname{ess} \sup }|\mu(w)|<\infty\right\} \\
\mathscr{B}(\Omega)=\{g(w): g \text { is holomorphic in } \Omega, \\
\left.\qquad|| g \|_{\Omega}=\iint_{\Omega}|g(w)| d u d v<\infty\right\} \\
\mathscr{N}(\Omega)=\left\{\mu(w): \mu \in \mathscr{K}(\Omega), \iint_{\Omega} \mu(w) g(w) d u d v=0 \text { for all } g \in \mathscr{B}(\Omega)\right\} .
\end{gathered}
$$

Transforming $\Omega$ onto $U$ by a conformal mapping $z \leftrightarrow w$ we find that if

$$
\varkappa(z) \frac{\overline{d z}}{d z}=\mu(w) \frac{\overline{d w}}{d w}, \quad f(z) d z^{2}=g(w) d w^{2},
$$

then $x \in \mathscr{K}(U)$ if and only if $\mu \in \mathscr{K}(\Omega)$, and $f \in \mathscr{B}=\mathscr{B}(U)$ if and only if $g \in \mathscr{B}(\Omega)$.

3 Refinements of (1.9) in which the measure of the set

$$
\left\{z: \varrho<|z|<1,|x(z)|>\|\varkappa\|_{\infty}-\delta\right\}
$$

is estimated in terms of $\delta$ from below are also possible. 
If $\Omega_{1} \subset \Omega_{2}$ then clearly $\mathscr{B}\left(\Omega_{1}\right) \supset \mathscr{B}\left(\Omega_{2}\right)$. Hence, if $x \in \mathscr{N}\left(\Omega_{1}\right)$, then

$$
\hat{x}(z)=\left\{\begin{array}{ll}
x(z), & z \in \Omega_{1} \\
0, & z \in \Omega_{2} \backslash \Omega_{1}
\end{array} .\right.
$$

will belong to $\mathscr{N}\left(\Omega_{2}\right)$. If we choose $\Omega_{1} \subset U=\Omega_{2}$ then (1.10) generates an element of $\mathscr{N}$. This remark will be used in Section 3.

In the case of a strip

$$
\Sigma=\left\{z: x+i y, y_{1}<y<y_{2}\right\}
$$

a useful criterion for membership in $\mathscr{N}(\Sigma)$ is as follows.

$\mathrm{L}$ e m m a 1.1. Let $v(z)=h_{0}(y), y_{1}<y<y_{2}$ be a complex bounded measurable function of the single variable $y, y=\operatorname{Im} z$. Then

$$
\nu \in \mathscr{N}(\Sigma) \Leftrightarrow \int_{y_{1}}^{y_{2}} h_{0}(y) d y=0 .
$$

Proof. If $f \in \mathscr{B}(\Sigma)$ then

$$
\int_{-\infty}^{\infty}|f(x+i y)| d x<\infty, \quad \text { for almost all } y, y_{1}<y<y_{2},
$$

and there exist sequences, $x_{n} \uparrow \infty, x_{n}^{\prime} \downarrow-\infty$, such that

$$
\int_{y_{1}}^{y_{2}}\left|f\left(x_{n}+i y\right)\right| d y \rightarrow 0, \quad \int_{y_{1}}^{y_{2}}\left|f\left(x_{n}^{\prime}+i y\right)\right| d y \rightarrow 0 .
$$

By Cauchy's theorem there therefore exists a constant $C_{f}$ such that

$$
\int_{-\infty}^{\infty} f(x+i y) d x=C_{f}, \text { a.a.y. }
$$

Therefore,

$$
\int_{\Sigma} \int f(z) h_{0}(y) d x d y=C_{f} \int_{y_{1}}^{y_{2}} h_{0}(y) d y
$$

Since there exist $f \in \mathscr{B}(\Sigma)$ for which $C_{f} \neq 0$, (e.g. for $f(z)=e^{-z^{2}}, C_{f}=$ $\sqrt{\pi}$ ) the result follows.

2. Extension inward and the class $\mathscr{N}_{\partial}$. In contrast with the situation described in Theorem 1.1 a function of class $\mathscr{N}$ cannot be prescribed 
arbitrarily in an annulus $\{\varrho<|z|<1\}$. Let $\beta(z)$ be a bounded, measurable, complex valued function, $\varrho<|z|<1$,

$$
b_{n}=\int_{\varrho<|z|<1} \beta(z) z^{n} d x d y, \quad n=0,1,2, \ldots .
$$

If there exists a function $\alpha(z),|z|<\varrho$ such that (1.3) belongs to $\mathscr{N}$ we say that extension inward of $\beta(z)$ is possible. The following two theorems give different types of growth conditions on $\left\{b_{n}\right\}$ related to this question.

Th e o r e m 2.1. A necessary condition on $\beta(z), \varrho<|z|<\mathrm{J}$, for extension inward to a function of class $\mathscr{N}$ is that

$$
\varlimsup_{n \rightarrow \infty}\left|b_{n}\right|^{1 / n} \leqq \varrho .
$$

A sufficient condition is that

$$
\varlimsup_{n \rightarrow \infty}\left|b_{n}\right|^{1 / n}<\varrho .
$$

Proof. If extension inward is possible

$$
b_{n}=-\int_{|z|<\varrho} \alpha(z) z^{n} d x d y, \quad n=0,1,2, \ldots .
$$

Hence $\left|b_{n}\right| \leqq \pi \varrho^{n} \sup _{|z|<\varrho}|\alpha(z)|$. This gives the necessary condition (2.1).

On the other hand, if (2.2) holds, then defining

$$
\alpha\left(r e^{i \vartheta}\right)=-\sum_{n=0}^{\infty} \frac{n+2}{2 \pi \varrho^{n+2}} b_{n} e^{-i n \vartheta}, \quad 0 \leqq r<\varrho, \quad 0 \leqq \vartheta<2 \pi,
$$

we obtain a continuous bounded function $\alpha(z)$ such that (1.3) satisfies $(1.2)$.

Alternatively, we also have the following conditions.

Th e o r e m 2.2. A necessary condition on $\beta(z), \varrho<|z|<1$, for extension inward to a function of class $\mathcal{N}$ is that

$$
\sum_{n=1}^{\infty} n \frac{\left|b_{n}\right|^{2}}{\varrho^{2 n}}<\infty \text {. }
$$

A sufficient condition is that

$$
\sum_{n=1}^{\infty} n \frac{\left|b_{n}\right|}{\varrho^{n}}<\infty
$$

Proof. We have already seen, above, that (2.5) is sufficient. To show that (2.4) is necessary, let

$$
f(z)=\sum_{k=0}^{n} \alpha_{k} z^{k}
$$


Then

$$
\begin{gathered}
\sum_{k=0}^{n} a_{k} b_{k}=\int_{\varrho<|z|<1} \int_{\mid<1} \beta(z) f(z) d x d y=-\int_{|z|<\varrho} \int_{k=0} \alpha(z) f(z) d x d y, \\
\left|\sum_{k}^{n} a_{k} b_{k}\right|^{2} \leqq \int_{|z|<\varrho}|\alpha(z)|^{2} d x d y \iint_{|z|<\varrho}|f(z)|^{2} d x d y \\
\leqq \pi^{2} \varrho^{2}|| \nu||_{\infty}^{2} \sum_{k=0}^{n} \frac{\left|a_{k}\right|^{2}}{k+1} \varrho^{2 k+2} .
\end{gathered}
$$

Choosing $a_{k}=\left((k+1) / \varrho^{2 k+2}\right) \overline{b_{k}}$ the above becomes

$$
\sum_{k=0}^{n} \frac{(k+1)\left|b_{k}\right|^{2}}{\varrho^{2 k}} \leqq \pi^{2} \varrho^{4}\|v\|_{\infty}^{2},
$$

which implies (2.4).

Example 2.1. $\beta(z)=(R-\bar{z})^{-1}, \varrho<|z|<1$, where $R$ is a fixed number, $R>1$. Here

$$
b_{n}=\frac{\pi\left(1-\varrho^{2 n+2}\right)}{(n+1) R^{n+1}}, \quad n=0,1,2, \ldots .
$$

By Theorem 2.2, inward extension of $\beta(z)$ to a function $v(z) \in \mathscr{N}$ is possible if and only if $\varrho R>1$.

Example 2.1 shows that local smoothness properties are not enough to guarantee an inward extension from a fixed annulus $\varrho<|z|<1$. It turns out, however, that our general objectives will not be compromised if inward extension is formulated in terms of a $\varrho$ that is not a-priori fixed. Accordingly a function $\beta(z)$ defined in some neighborhood $\left\{\varrho_{\beta}<|z|<1\right\}$ of $\partial U$ will be said to belong to the class $\mathscr{N}_{\partial}$ if there exists a function $v \in \mathscr{N}$ and a number $t_{\beta}, \varrho_{\beta}<t_{\beta}<1$, such that

$$
v(z)=\beta(z), \quad t_{\beta}<|z|<1 .
$$

It turns out that if $\beta(z)$ is sufficiently smooth near $\partial U$ then $\beta(z)$ will belong to $\mathscr{N}_{\partial}$. More precisely, a somewhat crude sufficient condition is the following.

Th e o r e $\mathrm{m}$ 2.3. If

$$
\sum_{n, n=0}^{\infty}\left|a_{m n}\right| R^{m+n}<\infty
$$

for some $R>1$, then the function

belongs to $\mathscr{N}_{\partial}$.

$$
\beta(z)=\sum_{m, n=0}^{\infty} a_{m n} z^{m} z^{n}, \quad \varrho<|z|<1,
$$


Proof. We have

$$
\begin{array}{r}
\sum_{m=0}^{\infty}\left|a_{m, m+k}\right| R^{2 m+k}=\sum_{\substack{0 \leqq m<\infty \\
n=m+k}}\left|a_{m n}\right| R^{m+n} \leqq \sum_{m, n=0}^{\infty}\left|a_{m n}\right| R^{m+n}=M<\infty, \\
k=0,1,2, \ldots .
\end{array}
$$

Hence,

$$
\sum_{m=0}^{\infty}\left|\alpha_{m, m+k}\right| \leqq \sum_{m=0}^{\infty}\left|\alpha_{m, m+k}\right| R^{2 m} \leqq \frac{M}{R^{k}}, \quad k=0,1,2, \ldots .
$$

For any $t, \varrho<t<1$, we have

$$
b_{k}=\int_{t<|z|<1} \int_{<1} \beta(z) z^{k} d x d y=\pi \sum_{m=0}^{\infty} a_{m, m+k} \frac{1-t^{2 m+2 k+2}}{m+k+1}, \quad k=0,1,2, \ldots
$$

Thus,

$$
\left|b_{k}\right| \leqq \pi \sum_{m=0}^{\infty}\left|a_{m, m+k}\right| \leqq \frac{M}{R^{k}}, \quad k=0,1,2, \ldots
$$

If we choose $t>1 / R$ then condition (2.2) is satisfied, and an inward extension of $\beta(z)$ from $\{t<|z|<1\}$ to a function of class $\mathscr{N}$ exists.

Following an important idea of K. Strebel [11] (formulated by him for the corresponding problem for quasiconformal mappings) the introduction of $\mathscr{N}_{\partial}$ allows the statement of a simple sufficient condition on $x$ which implies that $x \in \mathscr{K}_{T}$.

Th e o r e m 2.4. If $x \in \mathscr{K}$ and if there exists a $\tau$ such that $x-\tau \in \mathscr{N}_{\partial}$, $\|\tau\|_{\infty}<\left\|L_{\varkappa}\right\|$, then $\left\|L_{\varkappa}\right\|=L_{\varkappa}\left[\varphi_{0}\right]$ for some $\varphi_{0} \in \mathscr{B},\left\|\varphi_{0}\right\|=1$. In case $x \in \mathscr{K}^{*}$, then $x \in \mathscr{K}_{T}$.

Proof. We start with a preliminary observation [11]: Suppose $x$ has an extremal sequence $\left\{\varphi_{n}\right\}, \varphi_{n} \rightarrow \varphi_{0}$ loc.unif., ||$\varphi_{0} \|<1$. Then $x$ has a degenerative extremal sequence. If ||$\varphi_{0}||=0$ there is nothing to prove. If ||$\varphi_{0}||>0$, then, by Lemma 0.3 ,

$$
L_{x}\left[\varphi_{n}-\varphi_{0}\right]=L_{\varkappa}\left[\varphi_{n}\right]-L_{\varkappa}\left[\varphi_{0}\right] \rightarrow\left\|L_{\varkappa}\right\|-\left\|L_{\varkappa}\right\| \cdot\left\|\varphi_{0}\right\| .
$$

On the other hand, $\left\|\varphi_{n}-\varphi_{0}\right\| \rightarrow 1-\left\|\varphi_{0}\right\|$. Hence

$$
f_{n}=\frac{\varphi_{n}-\varphi_{0}}{\left\|\varphi_{n}-\varphi_{0}\right\|}
$$

is Strebel's degenerative extremal sequence.

Choose $v \in \mathscr{N}$ such that

$$
v(z)=\varkappa(z)-\tau(z), \quad \varrho<|z|<1 .
$$


If $\varkappa$ had a limit function $\varphi_{0},\left\|\varphi_{0}\right\|<1$, then if $f_{n}$ is formed as above,

$$
\begin{aligned}
L_{x}\left[f_{n}\right]=L_{\varkappa-\nu}\left[f_{n}\right] & =\int_{|z|<\varrho} \int_{\varrho \mid \varrho}(x-v) f_{n} d x d y+\int_{\varrho<|z|<1} \int_{\varrho<|z|<1} \tau f_{n} d x d y \\
& =\int_{0} \tau f_{n} d x d y+o(1) .
\end{aligned}
$$

So we have

$$
L_{\varkappa}\left[f_{n}\right] \rightarrow\left\|L_{\varkappa}\right\|, \quad \varlimsup_{n \rightarrow \infty}\left|L_{\varkappa}\left[f_{n}\right]\right| \leqq\|\tau\|_{\infty},
$$

a contradiction. Thus we conclude that $\left\|\varphi_{0}\right\|=1$ for every limit function $\varphi_{0}$ of $\varkappa$. By $(0.9), L_{\varkappa}\left[\varphi_{0}\right]=\left\|L_{\varkappa}\right\|$. If $\varkappa \in \mathscr{K}^{*}$, this implies

$$
\varkappa(z)=k \frac{\overline{\varphi_{0}(z)}}{\left|\varphi_{0}(z)\right|} .
$$

\section{Some examples}

Example 3.1. $\chi_{1}(z)=\chi_{S}(z)+\gamma(z)\left[1-\chi_{S}(z)\right], z \in U$, where $\chi_{S}$ is the characteristic function of the set $S, S \subset U$, meas $S>0$, and where

$$
\sup _{z \in A}|\gamma(z)|<1
$$

for some annulus $A=\{\varrho<|z|<1\}$. We will prove that

$$
\varkappa_{1} \in \mathscr{K}^{*} \Leftrightarrow \operatorname{meas}(U \backslash S)=0 \Leftrightarrow \varkappa_{1}(z) \equiv 1 .
$$

Suppose $\varkappa_{1} \in \mathscr{K}^{*}$. Then $\left\|L_{\varkappa_{1}}\right\|=\left\|\varkappa_{1}\right\|_{\infty}=1$. Let $\left\{\varphi_{n}\right\}$ be an extremal sequence for $\varkappa_{1}$. By (1.9), meas $(S \cap A)>0$. By (0.8),

$$
\begin{gathered}
\lim _{n \rightarrow \infty} \int_{(U \backslash S) \cap A} \int_{S \backslash A}\left|\varphi_{n}(z)\right| d x d y=0 \\
\lim _{n \rightarrow \infty}\left[\int_{S \cap A} \int_{A_{n}} \varphi_{n}(z) d x d y-\iint_{S \cap A}\left|\varphi_{n}(z)\right| d x d y\right]=0 .
\end{gathered}
$$

If $x_{1} \notin \mathscr{K}_{T}$ then $\left\{\varphi_{n}\right\}$ degenerates. In this case (3.2) implies that $\iint_{S \cap_{A}}\left|\varphi_{n}\right| d x d y \rightarrow 1$, and hence $\iint_{S \cap A} \varphi_{n}(z) d x d y \rightarrow 1$, and hence $\iint_{A} \varphi_{n}(z) d x d y \rightarrow 1$. But, by Cauchy's formula,

$$
\iint_{A} \varphi_{n}(z) d x d y=\pi\left(1-\varrho^{2}\right) \varphi_{n}(0),
$$


where $\varphi_{n}(o) \rightarrow 0$ since $\left\{\varphi_{n}\right\}$ degenerates. We have arrived at a contradiction, and must therefore conclude that $x \in \mathscr{K}_{T}$. Since meas $S>0$ this is possible only if $x(z) \equiv 1$.

If

$$
\varkappa_{1}(z)=\chi_{S}(z), \quad S=\left\{\varrho_{1}<|z|<\varrho_{2}\right\},
$$

|| $L_{\varkappa_{1}} \|$ can easily be calculated. Namely, we have

$$
L_{\varkappa_{1}}[f]=\iint_{S} f(z) d x d y=\left(\varrho_{2}^{2}-\varrho_{1}^{2}\right) \int_{U} \int f(z) d x d y, \quad f \in \mathscr{B},
$$

and therefore

$$
\left|L_{\varkappa_{1}}[f]\right| \leqq\left(\varrho_{2}^{2}-\varrho_{1}^{2}\right)\|f\| .
$$

On the other hand, $\left|L_{\varkappa_{1}}(1 / \pi)\right|=\varrho_{2}^{2}-\varrho_{1}^{2}$. Hence, for case (3.3),

$$
\left\|L_{\varkappa_{1}}\right\|=\frac{1}{\pi} \operatorname{meas} S .
$$

Example 3.2. As a prototype of a problem where the argument of Example 3.1 fails we consider

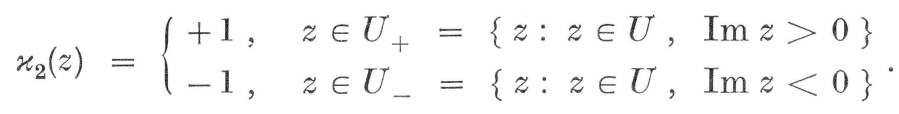

Using the connection with quasiconformal mapping, Strebel and the author have shown ${ }^{4}$ by studying the dilatations of appropriately chosen mappings that

$$
\left\|L_{\varkappa_{2}}\right\|<1 .
$$

We will now give an analytic proof ${ }^{5}$ of (3.6) using Lemma 0.1 and the methods of Section 1.

To prove (3.6) by means of Lemma 0.1 it is sufficient to find a function $v \in \mathscr{N}$ such that

$$
\operatorname{Re} v(z) \geqq \delta>0, \quad\left(z \in U_{+}\right), \quad \operatorname{Re} v(z) \leqq-\delta, \quad\left(z \in U_{-}\right),
$$

for with such a $v$ we get $\left\|\varkappa_{2}-t v\right\|_{\infty}<1$ when $t>0$ is sufficiently small.

(a) Let

$$
v_{0}(z)=-i e^{i \vartheta}=\sin \vartheta-i \cos \vartheta, \quad z \in U .
$$

${ }^{4}$ An outline of the proof may be found in the expository notes [5].

${ }^{5}$ Another analytic proof has recently been found by A. Harrington and M. Ortel who kindly allowed me to see their manuscript. 
By (1.1), $v_{0} \in \mathscr{N}$. Note that $\operatorname{Re} v_{0}(z) \geqq 0$ for $z \in U_{+}$, and $\operatorname{Re} v_{0}(z) \leqq 0$ for $z \in U_{-}$so that (3.7) is "almost" satisfied.

(b) Let $Q_{k}$ denote the $k^{\text {th }}$ quadrant of $U, k=1,2,3,4$. Let $w=\Phi(z)$ map $Q_{2}$ conformally onto

$$
\Sigma=\left\{w: 0<\operatorname{Im} w<\frac{\pi}{2}\right\}, \quad \text { with } \Phi(-1)=-\infty, \Phi(0)=+\infty .
$$

If we choose a bounded function $h(v),(v=\operatorname{Im} w)$, such that

$$
\int_{0}^{\pi / 2} h(v) d v=0
$$

then, by Lemma 1.1, $h \in \mathscr{N}(\Sigma)$. Therefore

$$
v_{2}(z)=\frac{\overline{\Phi^{\prime}(z)}}{\Phi^{\prime}(z)} h(v)=e^{2 i \Theta(z)} h(v) \in \mathscr{N}\left(Q_{2}\right), \quad(v=\operatorname{Im} \Phi(z)) .
$$

By considering the boundary behavior of $\Phi, \Phi^{\prime}$ at $z=-1, z=0$, the following is easily verified:

$$
\begin{aligned}
e^{2 i \Theta(z)} & =\frac{z}{\bar{z}}+o(1)=e^{-2 i v}+o(1), \quad \text { as } z \rightarrow 0, z \in Q_{2}, \\
e^{2 i \Theta(z)}= & \frac{z+1}{\overline{z+1}}+o(1)=e^{2 i v}+o(1), \quad \text { as } z \rightarrow-1, z \in Q_{2},
\end{aligned}
$$

Choosing

$$
h(v)=e^{-2 i v}+i, \quad 0<v<\frac{\pi}{2},
$$

we therefore find, for $z \in Q_{2}, v=\operatorname{Im} \Phi(z)$,

$v_{2}(z)=\left\{\begin{array}{l}e^{-4 i v}+i e^{-2 i v}+o(1), \quad z \rightarrow 0, \\ 1+i e^{2 i v}+o(1), \quad z \rightarrow-1, \\ 1+i+o(1), \quad z \rightarrow x_{0}, \text { uniformly on every compact subset of } R_{3} .\end{array}\right.$

Due to the conformality of $e^{\Phi(z)}$ at $z=0$,

$$
z \rightarrow 0 \Rightarrow \frac{v}{\arg z} \rightarrow 1 \text {, uniformly with respect to } \arg z \text {. }
$$

Thus there exists an $\varepsilon>0$ such that

$$
z \in Q_{2}, \quad \pi-\varepsilon<\arg z<\pi \Rightarrow \operatorname{Re} v_{2}(z) \geqq \delta^{\prime}>0 .
$$


Next, we extend $v_{2}(z)$ to all of $U$ by defining it as 0 in $U \backslash Q_{2}$. The resulting function belongs to $\mathscr{N}$.

(c) We define the sister functions

$$
v_{4}(z)=-v_{2}(-z), \quad v_{1}(z)=\overline{-v_{4}(\bar{z})}, \quad v_{3}(z)=\overline{-v_{2}(\bar{z})} .
$$

Since $v_{2} \in \mathscr{N}$ it is clear from (1.1) that $v_{k} \in \mathscr{N}, k=1,3,4$, also. The function

$$
\sum_{k=1}^{4} v_{k}(z), \quad z \in U,
$$

therefore belongs to $\mathscr{N}$ and displays the desired behavior (3.7) in the angular set

$$
-\varepsilon<\arg z<\varepsilon, \quad \pi-\varepsilon<\arg z<\pi+\varepsilon,
$$

symmetric with respect to the real axis in $U$. Therefore, if we choose $c>0$ sufficiently small, the function

$$
v(z)=v_{0}(z)+c \sum_{k=1}^{4} v_{k}(z), \quad z \in U,
$$

which also belongs to $\mathscr{N}$, will satisfy (3.7).

Remark. The same function $v(z)$ satisfying (3.7) evidently serves to determine a considerably more general class of $\varkappa^{\prime} s$, other than just $\varkappa_{2}$, which belong to $\mathscr{K} \backslash \mathscr{K}^{*}$.

\section{References}

[1] AHLFORS, L. V.: Some remarks on Teichmüller's space of Riemann surfaces. - Ann. of Math. (2) 74, 1961, 171-191.

[2] HAMm,TON, R. S.: Extremal quasiconformal mappings with prescribed boundary values. - Trans. Amer. Math. Soc. 138, 1969, 399-406.

[3] REICH, E.: On the extremality and unique extremality of affine mappings. - Topics in analysis. Colloquium on mathematical analysis, Jyväskylä 1970. Lecture Notes in Mathematics 419. Springer-Verlag, BerlinHeidelberg-New York, 1974, 294-304.

[4] - - On the relation between local and global properties of boundary values for extremal quasiconformal mappings. - Discontinuous groups and Riemann surfaces. Proceedings of the 1973 conference at the University of Maryland. Annals of Mathematics Studies 79. Princeton University Press and University of Tokyo Press, Princeton, New Jersey, 1974, $391-407$.

[5] -»- Quasiconformal mappings of the disk with given boundary values. - Advances in complex function theory. Proceedings of seminars held at Maryland University 1973/74. Lecture Notes in Mathematics 505. Springer-Verlag, Berlin-Heidelberg-New York, 1976, 101-137. 
[6] REICH, E., and K. STREBEL: On the extremality of certain Teichmüller mappings. - Comment. Math. Helv. 45, 1970, 353-362.

[7] -»- - - Extremal quasiconformal mappings with given boundary values. - [Notes] ETH Zürich, Forschungsinstitut für Mathematik, 1972, $1-43$.

[8] - - - - Extremal quasiconformal mappings with given boundary values. - Contributions to analysis. A collection of papers dedicated to Lipman Bers. Academic Press, New York-London, 1974, 375-391.

[9] STREBEL, K.: Zur Frage der Eindeutigkeit extremaler quasikonformer Abbildungen des Einheitskreises. - Comment. Math. Helv. 36, 1962, 306-323.

[10] -»- Zur Frage der Eindeutigkeit extremaler quasikonformer Abbildungen des Einheitskreises II. - Comment. Math. Helv. 39, 1964, 77-89.

[11] - - - On quadratic differentials and extremal quasi-conformal mappings. - Proceedings of the International Congress of Mathematicians, Vancouver 1974, vol. 2. Canadian Mathematical Congress, 1975, 223-227.

[12] TEICHMÜLLER, O.: Extremale quasikonforme Abbildungen und quadratische Differentiale. - Abh. Preuss. Akad. Wiss. Math.-Nat. Kl. 1939: 22, $1-197$.

University of Minnesota

Department of Mathematics

Minneapolis, Minnesota 55455

USA

Received 9 April 1975 